\title{
Breast and gut: the relationship between lactating mammary function and neonatal gastrointestinal function
}

\author{
BY LAWRENCE T. WEAVER \\ MRC Dunn Nutrition Unit, Milton Road, Cambridge CB4 1 XJ
}

\section{HYPOTHESIS}

Throughout the class mammalia there is an intimate relationship between lactating mammary function and neonatal gastrointestinal function. The extent of the contribution of breast milk to the adaptation of the newborn to extrauterine life is related inversely to the time after conception at which birth occurs in the course of gastrointestinal development. Milk is nutrients packaged for transfer from mother to young, but also contains a range of non-nutrient factors which assist perinatal adaptation. The neonatal gut is adapted not only for the utilization of nutrients provided by milk, but also depends on non-nutrient factors to compensate for its immature digestive and barrier functions, and trophic and other factors to regulate the transition from intrauterine to extrauterine life. During mammalian evolution there has been a trend towards birth later in the course of gastrointestinal development with a consequent reduction in the range of biological functions of milk feeding. This is reflected in the structure and function of the neonatal gut.

\section{THE MAMMALIAN GUT}

The feeding of the young on milk is a characteristic of all mammals, and one that gives the class its name. Mammals have been successful in exploiting a wide range of nutritional habitats: the variety of diets they consume allows them to occupy ecological niches as varied as the skies, tree-tops, oceans and plains. The foods of mammals range from insects to meat to fruit to foliage.

This dietary diversity is reflected in differences in structure and function of the gastrointestinal tract. In all mammals such variations occur on a common design evolved first by their cordate ancestors some 500 million years ago. The gut of the carnivore is short and tubular, adapted for the utilization of a compact, nutrient-dense diet. That of the herbivore is long, and has a capacious fore or hind stomach for the microbial digestion of vegetable matter (Chivers \& Hladic, 1980). Just as adult mammals have evolved guts which suit them to their nutritional niches, so too have their young evolved guts to suit their peculiar postnatal requirements: milk feeding.

\section{PLACENTA, BREAST AND GUT}

The ecological niche of the fetus is the uterus where, suspended in amniotic fluid, it enjoys a secure relationship with its mother via the placenta. The circulation of this fluid, which contains enzymes, immunoglobulins, hormones and trophic factors, may help to prepare the gastrointestinal epithelium for its postnatal task (Mulvihill et al. 1986).

The placenta is an organ for the exchange of nutrients, gases, heat and other substances between mother and fetus during prenatal life. Its structure varies widely among mammals, but morphology gives no clue to phylogeny. The transport function of 
the placenta, particularly in relation to transfer of immunoglobulins and other physiologically active substances, is inversely related to the capacity of the neonatal gut to absorb and process such macromolecules.

The mammalian placenta has been defined as any intimate apposition of fetal organs to maternal tissues for physiological exchange (Mossman, 1937). At birth the newborn mammal exchanges secure dependence on a ready and regulated supply of nutrients and other factors via the placenta for the intermittent supply of nutrients and non-nutrient factors from the breast in the form of colostrum and milk. The ecological niche of the neonate is the lactating mammary gland; until weaning it remains dependent on a fluid of highly regulated composition for the utilization of which its gut is uniquely adapted. The suckling neonate may, therefore, be considered an 'extra-gestate' fetus in which major functions of the placenta are undertaken by the breast and gut.

\section{THE LACTATING MAMMARY GLAND}

Despite marked differences between species in the relative size, number and position, of mammary glands, their anatomical and histological structure is relatively uniform. They comprise secretory and ductal tissue, supported by fat and connective tissue whence colostrum and milk are synthesized and secreted. Milk consists of nutrients and other substances packaged for transfer from mother to baby and, like the closely opposed membranes of the placental barrier, the secretory and absorptive epithelia of the breast and gut are complementary in structure and function (Fig. 1).

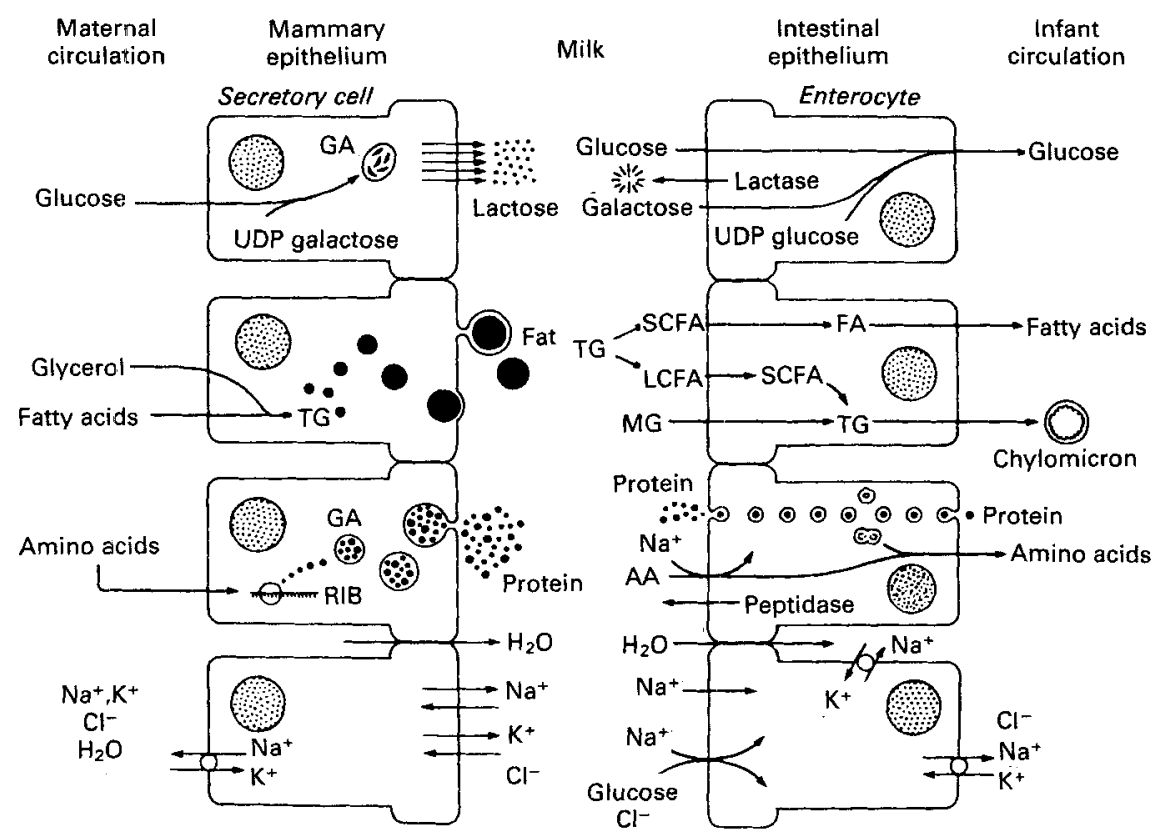

Fig. 1. The complementary structure and function of the lactating mammary epithelium and neonatal absorptive epithelium illustrating the synthesis, transfer as milk, utilization and absorption of the major nutrients. GA, golgi apparatus; RIB, ribosomes; TG, triacylglycerols; SCFA, short-chain fatty acids; LCFA, long-chain fatty acids; MG, monoacylglycerols; FA, fatty acids; AA, amino acids. 
It has been argued that the composition of mammalian milk is a reflection of the environmental conditions, litter size, postnatal nutrient requirements, and rates of basal metabolism and growth of the newborn (Oftedal, 1984). The milk of the seal, which requires fat for energy and insulation, has a high lipid content ( $490 \mathrm{~g} / \mathrm{l})$, while the newborn shrew, which doubles its body-weight in $24 \mathrm{~h}$, has a milk rich in protein $(10 \mathrm{~g} / 1)$; in both cases about ten times the concentrations of these nutrients in the milk of the newborn baby, which is born with ample fat stores and has a relatively slow growth rate (Widdowson, 1981).

The simple correlation of milk nutrient composition with postnatal nutritional needs is insufficient to explain the wide range of neonatal suckling strategies that mammals have evolved to feed their young. Mammary glands may be related to the highly vascularized brooding spots on the breasts of birds, used to hatch eggs. It has also been suggested that they evolved from sebaceous (apocrine) glands in the skin of the reptilian ancestors (therapsids) of mammals during the Mesozoic era around 200 million years ago. The protolacteal secretions of the glands of these early mammals may have served to attach eggs, annoint them with a protective coating or afforded their young immunological defence against microbial infection (Long, 1969). The milk of presentday mammals has constituents other than nutrients which assist in perinatal adaptation to enteral feeding, provide mucosal protection and compensate for deficiencies and immaturities of neonatal digestive and absorptive function.

\section{THE NEONATAL GUT}

The mammalian gastrointestinal tract develops in six stages (Weaver, 1991). An embryological stage of infolding of the endodermal layer to form the gut tube, is followed by organogenesis when the major organs of the digestive system are formed. After this there is a stage of cytodifferentiation when the distinct cell types of the gastrointestinal epithelium become recognizable. This is followed by a stage of growth and maturation of the gut and its mucosa in preparation for the task of independently feeding the newborn. The neonatal stage of adaptation to enteral nutrition is succeeded by a weaning stage of transition from milk to solid diet, when breast feeding gradually ceases. This is followed by an adult stage of mature gut function, and may be concluded by a stage of waning digestive function during senescence.

The neonatal gut has several major functions. It is an organ of nutrition, with digestive, absorptive, secretory and motile functions adapted to a milk diet. It is also part of the immune system, containing both humoral and cellular elements of the gutassociated lymphoid tissue (GALT). It is a large and diffuse endocrine organ which secretes locally-acting 'gut hormones' or paracrines which may regulate intestinal and metabolic adaptation to extrauterine life (Aynsley-Green, 1985). It plays a part in water conservation and electrolyte homeostasis, and its large bowel is the home of a symbiotic microbial flora which in herbivores plays a central role in digestion.

\section{BREAST-GUT INTERACTIONS}

Birth is a movable event which in different mammals occurs at different time-points during the course of the development of the digestive system. The neonatal gastrointestinal tract of each species is adapted to its particular postnatal requirements, and 


$\begin{array}{lcccccc}\text { Cytodifferentiation } & & \text { Maturation-Perinatal Adaptation } & & \\ & & \uparrow & \uparrow & \uparrow & \uparrow & \uparrow \\ \text { Birth } & \text { Kangaroo } & \text { Rat } & \text { Cow } & \text { Man } & \text { Guinea-pig } \\ & & & & & & \\ \text { Nutrients } & + & + & + & + & + \\ \text { Digestive enzymes } & + & + & + & + & \\ \text { Protective factors } & + & + & + & & \text { Milk } \\ \text { Trophic factors } & + & + & & & \text { composition } \\ \text { Homeostatic factors } & + & & & & & \end{array}$

Fig. 2. The time of birth of five mammals in relation to the stages of development of the gastrointestinal tract. The contribution of the components of milk to neonatal adaptation are shown: the earlier that birth occurs during the course of gastrointestinal development, the greater the contribution of the constituents of milk to neonatal adaptation.

differs from that of the adult in its digestive, absorptive and barrier functions. During the evolution of viviparity from oviparity adverse environmental conditions and limited availability of food may have favoured the retention of the young with mother. The consequences of such a change in neonatal feeding strategy was the birth of altricial progeny after a shorter gestation, at an earlier stage in the course of gastrointestinal development, necessitating a specialized diet, namely milk (Weaver, 1986).

Such immature young require from milk a range of non-nutrient factors which include immunoglobulins, trophic factors, digestive enzymes and other physiologically active polypeptides and oligosaccharides. The mode of uptake, sorting and transport of such macromolecules by the neonatal gut is linked closely to the relative length of gestation, postnatal physiological needs and the composition of milk received by the newborn. Those species which bear young early in the course of gastrointestinal development (altricial) depend on colostrum and milk for non-nutrient factors which are transferred placentally in precocial species. Their small intestines retain the capacity for macromolecular uptake during the suckling period. The gut of precocial neonates, on the other hand, exhibits only a limited and brief period of macromolecular uptake (Weaver \& Walker, 1989).

Fig. 2 illustrates the stages of mammalian gut development, from cytodifferentiation to weaning, with the time of birth of five species shown in relation to these stages. The contributions of the components of milk to neonatal adaptation of each is shown.

1. Guinea-pig. The precocial guinea-pig is born late in the course of gastrointestinal development, after a gestation of $67 \mathrm{~d}$. At birth it is independent and mature, with hair, teeth, autonomous thermoregulation and locomotion, like a miniature adult. The neonatal guinea-pig may be reared on an artificial milk formula, weaned onto a solid diet soon after delivery (Weaver et al.1988), and even at peak lactation catabolizes its own body fat to supply a substantial portion of its energy needs.

2. Man. The human neonate consumes a milk containing essential nutrients, as well as protective, trophic and digestive factors. Birth is earlier in the course of gastrointestinal development than in the guinea-pig and calf, and the newborn baby has no teeth, is immobile without maternal help, and cannot cope with a solid diet until some months after birth. The non-nutritional components of breast milk may contribute to adaptation to extrauterine life, and even later health and development of the baby.

Breast milk is rich in secretory immunoglobulin A (sIgA). Secreted by the lactating mammary gland, sIgA resists gastric hydrolysis, and is capable of survival during passage 
through the gastrointestinal tract (Prentice et al. 1987). Together with lactoferrin, sIgA represents $30 \%$ of milk protein. It offers passive protection at the gastrointestinal epithelium where it acts to exclude foreign antigens. Through the enteromammary circulation mother may confer passive protection against those antigens to which her own gastrointestinal tract has been exposed (Kleinman \& Walker, 1979). Antigens ingested by mother are recognized and sampled at $\mathbf{M}$ cells within Peyer's patches in the small intestine. Primed plasma cells then pass via the lymphatic and venous circulation to her breast where specific sIgA is synthesized, secreted in milk and ingested by the suckling infant. SIgA and other factors in human milk may protect the newborn, particularly those born prematurely and in the developing world (Briend et al. 1988) from gastrointestinal disease.

Breast milk also contains trophic factors, hormones, cytokines and other regulatory peptides (Koldovsky \& Thornburg, 1987). Of the trophic factors active in the newborn, epidermal growth factor (EGF) is the best studied. It is a small polypeptide with mitogenic, anti-secretory and cytoprotective properties. Its presence in amniotic fluid (Weaver et al. 1989) and colostrum (Read et al. 1984) suggests that it plays a part in perinatal adaptation to extrauterine nutrition and gastrointestinal function (Weaver \& Walker, 1988); to activate mucosal function, to diminish gastric hydrolysis of potentially useful milk macromolecules, and to protect the gastrointestinal epithelium from digestion by endogenous and exogenous enzymes.

EGF receptors are found on enterocytes from the 19th week of gestation and may be responsible for mediating perinatal changes in gastrointestinal function. Small intestinal lactase ( $E C$ 3.2.1.108) activity rises slowly during the first and second trimesters of pregnancy, before a steep rise just before term (Antonowicz \& Lebenthal, 1977). Using organ culture to examine the effects of EGF on the function of the human fetal jejunum it has been shown that EGF causes induction of lactase and repression of sucrase (EC 3.2.1.48) activity (Menard et al. 1988). Acceleration of maturation of lactase activity may be particularly relevant to the preterm infant, exposed prematurely to milk feeds.

The newborn infant has an immediate requirement for fat, to provide energy, insulation, neural tissue and membrane synthesis. In the full-term infant adipose stores and breast milk satisfy this demand. Milk, in addition, contains lipases which may assist in the digestion of fat during a period of pancreatic and hepato-biliary hypofunction (Hamosh, 1982). Compared with the adult, in whom the pancreas is the major source of lipases, concentrations of pancreatic enzymes in the neonate are significantly depressed. In the preterm infant, pancreatic lipase is only $10 \%$ of that of the adult, and the bile-salt pool is only $50 \%$ of that found in the mature neonate (Watkins et al. 1975). Depressed pancreatic function ensures that the immature microvillus membrane is spared digestion by pancreatic proteolytic enzymes, and permits prolonged activity of essential brushborder enzymes, (such as lactase), and other physiologically active non-nutrient milk proteins, (such as $\operatorname{sigA}$ ), which might otherwise be digested. The improved fat absorption seen in neonates receiving fresh human milk probably resides in its specific bile-salt-stimulated lipase, which together with lingual lipase, accounts for the ability of the newborn to make full use of the fat in breast milk.

In conjunction with immature digestive function, the greater passive permeability of the immature intestine to both micro- and macromolecules (Weaver et al. 1984; Jakobsson et al. 1986) may allow the passage of small quantities of ingested non-nutrient 
molecules across the gut mucosa. Although an 'apical tubular system', capable of pinocytosis of 'meconium corpuscles' exists in the human fetal small intestinal epithelium from about 12 to 22 weeks (Colony, 1983), postnatal macromolecular uptake does not appear to be an adaptation essential for neonatal survival. The human newborn may be reared on the modified milk of another species (cow) or even one of plant origin (soya bean) devoid of such macromolecules. While the neonatal gut is adapted to utilize the nutrients of human milk efficiently, it has negligible capacity to actively absorb physiological macromolecules, such as immunoglobulins and trophic factors. In species such as the rat, however, in which birth occurs at an earlier stage of gut development, the neonatal gastrointestinal tract retains a specialized system for the uptake, sorting and transport of macromolecules.

3. Calf. The calf is born relatively mature after a gestation of $280 \mathrm{~d}$. During the neonatal period ingested milk bypasses the rumen, reticulum and omasum (essential for microbial fermentation of foliage in the cow), via the oesophageal groove, to the abomasum. The newborn calf depends on milk for nutrients, and for protective immunoglobulins (principally IgG). The small intestine has the capacity to absorb them intact, whereafter they contribute to systemic immunity. Intestinal closure to macromolecular uptake occurs within $48 \mathrm{~h}$ of birth, in association with a decline in gastric acidity. Without colostrum the calf gets infective diarrhoea, fails to thrive and may die (Patt, 1977). Birth is earlier in the course of gastrointestinal development than in the guinea-pig.

4. Rat. The altricial rat is born hairless, blind, unable to control its body temperature or independently evacuate bowels or bladder. After a gestation of $21 \mathrm{~d}$ it is closely dependent on its dam not only for nutrients in milk, and protective factors, but also makes use of trophic factors in amniotic fluid (Weaver et al. 1990) and colostrum (Berseth, 1987) to promote development of the intestinal mucosa. The stage of cytodifferentiation occurs during the last $3-4 \mathrm{~d}$ before birth, and the small intestine of the newborn rat has the capacity to take up proteins intact; in the jejunum colostral IgG is transferred by receptor-mediated endocytosis across the mucosa into the circulation, and in the ileum EGF and other regulatory peptides are absorbed by non-selective pinocytosis (Weaver \& Walker, 1989).

It is likely that digestive enzymes in milk are particularly important in altricial species where they compensate for immature pancreatic function: lipase has been found in the milk of dogs and cats (Freed et al. 1986). Those species that depend on milk for both protective and trophic factors, and which have a small intestine adapted for their absorption, must limit endogenous digestive enzymes that might interfere with this process. Gastric acid and pepsinogen, and pancreatic proteases, are all very low during the first 2 weeks postnatally, and increase markedly towards weaning, to ensure that during the suckling period luminal digestion of milk proteins is minimized. Extracellular sorting is a feature of the mature gut and that of the precocial newborn. In the neonatal rat sorting of absorbed macromolecules takes place intracellularly, in specific organelles which determine what is digested, what is utilized within the cell, and what is allowed to pass through to the circulation intact (Weaver \& Walker, 1989). Birth of this altricial species is relatively early in the course of gastrointestinal development and the newborn is dependent on nutrient, protective, trophic and digestive components of breast milk.

5. Kangaroo. Marsupials have limited 'fetal immunological tolerance' and the joey, the 
highly immature progeny of the kangaroo, is born after a gestation of only 3 weeks, weighing $750 \mathrm{mg}$. It makes its way from the birth canal to the pouch, latching onto one of four teats where it remains for the succeeding 6 months (Tyndale-Biscoe, 1973). During this long period of dependence on milk it utilizes not only the nutrient and non-nutrient components of milk, but also what may be called 'homeostatic factors'. During extragestate development every aspect of the immature metabolism of the 'embryological' joey (such as water and electrolyte balance, energy homeostasis) must be regulated by hormones and other factors supplied by milk. Throughout this intra-pouch period the joey's lips are fused around the teat which extends into its pharynx creating an umbilical cord-like continuity between mother and baby. In marsupials breast and gut truely may be said to undertake the functions that in some eutherians the placenta would perform for an equal length of time.

\section{CONCLUSIONS}

The relationship between lactating mammary function and neonatal gastrointestinal function is an example of the parallel evolution of two organs, the breast and gut, which after birth, together undertake functions performed by the placenta during intrauterine life. During mammalian evolution the process of suckling has been adapted to serve a variety of different strategies for feeding the young, and a close interconnection of breast and gut has persisted. However, there has been a trend towards the occurrence of birth later in the course of gastrointestinal development with a consequent reduction in the range of biological functions of milk feeding. In altricial species non-nutrient factors in colostrum and milk make a major contribution to gastrointestinal function, defence and the regulation of metabolism. Deficient luminal digestion permits the survival of physiologically active macromolecules during passage through the gut, and its mucosa possesses a sophisticated system for their uptake, sorting, transfer and utilization. In precocial young, which have a lesser need for intact macromolecular uptake, gut development is almost complete by birth, such a system is not evident and breast milk is largely the sole source of nutrition. Such major differences between altricial and precocial young in digestive and absorptive capacities are also reflected in differences in maturity of motor function necessary to propel milk through the gut (Ruckebusch, 1986).

In man each non-nutrient factor makes up for a deficiency of neonatal gut function, which alone is insufficient to fully meet the demands of postnatal enteral nutrition (Fig. 3 ). In addition to lactose, the major carbohydrate which meets the energy requirements of the newborn, and for which the neonatal gut is uniquely adapted, breast milk contains lipase, to compensate for the immaturity of pancreatic function of the newborn. SIgA provides protection of an epithelium that is immature in its barrier function. Trophic factors such as EGF are involved in the maturation of the immature gut and help to regulate perinatal adaptation.

Breast and gut interact, not simply through the food produced at the one and utilized at the other, but also through non-nutritional messages that pass between the two. The neonatal feeding strategies of these five different mammalian examples show that if we are to understand the postnatal nutritional requirements and gastrointestinal function of the newborn, we must be aware not only of the nutrient and non-nutrient components of the milk it receives, but also of the stage to which its gut has developed at birth. 

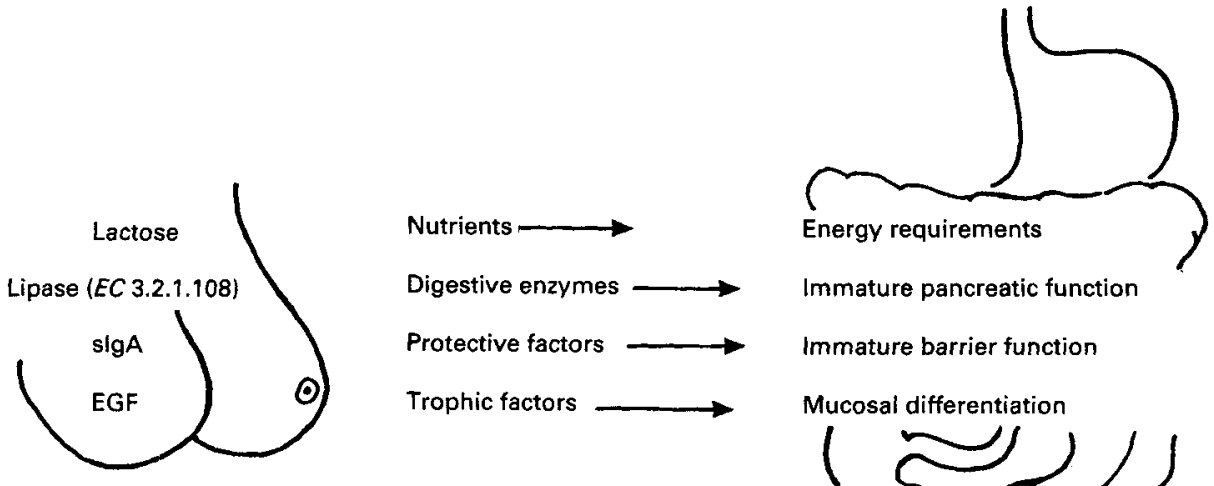

Immature pancreatic function

Immature barrier function

Mucosal differentiation

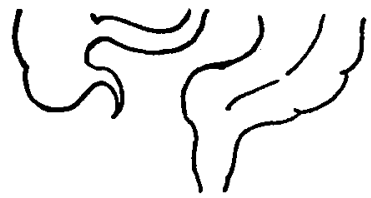

Fig. 3. Examples of the contribution of nutrients and non-nutrient factors to neonatal gastrointestinal adaptation in man. Each non-nutrient factor makes up for a deficiency or immaturity of perinatal digestive function. sIgA, secretory immunoglobulin A; EGF, epidermal growth factor.

\section{REFERENCES}

Antonowicz, I. \& Lebenthal, E. (1977). Developmental pattern of small intestinal enterokinase and disaccharidase activities in the human fetus. Gastroenterology 72, 1299-1303.

Aynsley-Green, A. (1985). Metabolic and endocrine interrelations in the human fetus and neonate. American Journal of Clinical Nutrition 41, 339-417.

Berseth, C. L. (1987). Enhancement of intestinal growth in neonatal rats by epidermal growth factor in milk. American Journal of Physiology 253, G662-665.

Briend, A., Wojtyniak, B. \& Rowland, M. G. M. (1988). Breast feeding, nutritional state, and child survival in rural Bangladesh. British Medical Journal 296, 879-882.

Chivers, D. J. \& Hadic, C. M. (1980). Morphology of the gastrointestinal tract in primates: comparison with other mammals in relation to diet. Journal of Morphology 166, 337-386.

Colony, P. C. (1983). Successive phases of human fetal gastrointestinal development. In Nutritional Adaptation of the Gastrointestinal Tract of the Newborn, pp. 3-28 [N. Kretchmer and A. Minikowski, editors]. New York: Raven Press.

Freed, L. M., York, C. M., Hamosh, M., Mehta, N. R., Sturman, J. A., Oftedal, O. T. \& Hamosh, P. (1986). Bile salt stimulated lipase: the enzyme is present in non-primate milk. In Human Lactation, pp. 596-601 [M. Hamosh and S. Goldman, editors]. New York: Plenum.

Hamosh, M. (1982). Lingual and breast milk lipases. Advances in Pediatrics 29, $22-67$.

Jakobsson, I., Lindberg, T., Lothe, L., Axelsson, I. \& Benediktsson, B. (1986). Human alpha-lactalbumin as a marker of macromolecular absorption. Gut 27, 1029-1034.

Kleinman, R. E. \& Walker, W. A. (1979). The enteromammary immune system. Digestive Disease and Science 24, 876-882.

Koldovsky, O. \& Thornburg, W. (1987). Hormones in milk. Journal of Pediatric Gastroenterology and Nutrition 6, 172-196.

Long, C. A. (1969). The origin and evolution of mammary glands. Bioscience 19, 519-523.

Menard, D., Arsenault, P. \& Pothier, P. (1988). Biologic effects of epidermal growth factor in human fetal jejunum. Gastroenterology 94, 656-663.

Mossman, H. W. (1937). Comparative morphogenesis of the fetal membranes and accessory uterine structures. Contributions to Embryology 158, 133-247.

Mulvihill, S. J., Stone, M. M., Fonkalsrud, E. W. \& Bebas, H. T. (1986). Trophic effect of amniotic fluid on fetal gastrointestinal development. Journal of Surgical Research 40, 291-296.

Oftedal, O. T. (1984). Milk composition, milk yield and energy output at peak lactation: a comparative review. Symposium of the Zoological Society of London 51, 33-85. 
Patt, J. A. (1977). Factors affecting the duration of intestinal permeability to macromolecules in newborn animals. Biological Reviews 52, 411-429.

Prentice, A., Ewing, G., Roberts, S. B., Lucas, A., MacCarthy, A., Jarjou, L. M. \& Whitehead, R. G. (1987). The nutritional role of breast milk IgA and lactoferrin. Acta Paediatrica Scandinavica 76, 592-598.

Read, L. C., Upton, F. M., Francis, G. L., Wallace, J. C., Dahlenberg, G. W. \& Ballard, F. J. (1984). Changes in growth-promoting activity of human milk during lactation. Pediatric Research 18, 133-138.

Ruckebusch, Y. (1986). Development of digestive motor patterns during perinatal life: mechanism and significance. Journal of Pediatric Gastroenterology and Nutrition 5, 523-536.

Tyndale-Biscoe, H. (1973). Life of Marsupials. London: Edward Arnold.

Watkins, J. B., Szczepanik, P., Gould, J. P., Klein, P. \& Lester, R. (1975). Bile salt metabolism in the human premature infant. Gastroenterology 69, 706-713.

Weaver, L. T. (1986). Milk and the neonatal gut: comparative lessons to be learnt. Equine Veterinary Journal $18,427-429$.

Weaver, L. T. (1991). Development of the gastrointestinal tract: anatomy and embryology. In Pediatric Gastrointestinal Disease, pp. 195-216 [W. A. Walker, P. R. Durie, J. R. Hamilton, J. A. Walter-Smith and J. B. Watkins, editors]. Toronto: Decker.

Weaver, L. T., Freiberg, E., Israel, E. J. \& Walker, W. A. (1989). Epidermal growth factor in human amniotic fluid. Gastroenterology 95, 1346.

Weaver, L. T., Gonnella, P. A., Israel, E. J. \& Walker, W. A. (1990). Uptake and transport of epidermal growth factor (EGF) by the small intestinal epithelium of the fetal rat. Gastroenterology 96, 828-837.

Weaver, L. T., Laker, M. F. \& Nelson, R. (1984). Intestinal permeability in the newborn. Archives of Disease in Childhood 59, 236-241.

Weaver, L. T., Landymore-Lim, A. E. N. \& Hudson, G. J. (1988). The guinea pig as a model for the study of the effects of milk on growth and development. Growth Development and Aging 52, 91-96.

Weaver, L. T. \& Walker, W. A. (1988). Epidermal growth factor and the developing human gut. Gastroenterology 94, 845-847.

Weaver, L. T. \& Walker, W. A. (1989). Uptake of macromolecules in the neonate. In Human Gastrointestinal Development, pp. 731-748 [E. Lebenthal, editor]. New York: Raven Press.

Widdowson, E. M. (1981). Feeding the Newborn Mammal. Burlington, N.C.: Carolina Biology Readers. 